Article

\title{
The Study of Dynamical Potentials of Highly Excited Vibrational States of $\mathrm{HOBr}$
}

\author{
Aixing Wang ${ }^{1,2}$, Lifeng Sun ${ }^{3}$, Chao Fang ${ }^{3, *}$ and Yibao Liu ${ }^{1}$ \\ 1 Engineering Research Center of Nuclear Technology Application (East China Institute of Technology), \\ Ministry of Education, Nanchang 330013, China; E-Mails: xingxing_fz@sina.com (A.W.); \\ liuyb01@mails.tsinghua.edu.cn (Y.L.) \\ 2 College of Science, East China Institute of Technology, Nanchang 330013, China \\ 3 Institute of Nuclear and New Energy Technology, Tsinghua University, Beijing 100084, China; \\ E-Mail:1fsun@tsinghua.edu.cn
}

* Author to whom correspondence should be addressed; E-Mail: fangchao@tsinghua.edu.cn; Tel.: +86-10-6277-1140; Fax: +86-10-6277-1150.

Received: 24 December 2012; in revised form: 31 January 2013 / Accepted: 8 February 2013 / Published: 5 March 2013

\begin{abstract}
The vibrational nonlinear dynamics of $\mathrm{HOBr}$ in the bending and $\mathrm{O}-\mathrm{Br}$ stretching coordinates with anharmonicity and Fermi 2:1 coupling are studied with dynamical potentials in this article. The result shows that the $\mathrm{H}-\mathrm{O}$ stretching vibration mode has significantly different effects on the coupling between the $\mathrm{O}-\mathrm{Br}$ stretching mode and the $\mathrm{H}-\mathrm{O}-\mathrm{Br}$ bending mode under different Polyad numbers. The dynamical potentials and the corresponding phase space trajectories are obtained when the Polyad number is 27 , for instance, and the fixed points in the dynamical potentials of $\mathrm{HOBr}$ are shown to govern the various quantal environments in which the vibrational states lie. Furthermore, it is also found that the quantal environments could be identified by the numerical values of action integrals, which is consistent with former research.
\end{abstract}

Keywords: $\mathrm{HOBr}$; highly excited vibrational state; geometrical shape of dynamical potential; phase space trajectory 


\section{Introduction}

Atmospheric pollution has caused widespread concern because it has led to not only a lot of diseases but also serious damage to the ecological balance. $\mathrm{HOBr}$ is a very unstable oxidizing substance in the atmosphere. Radical generation of Bromine $(\mathrm{Br})$ is caused by the $\mathrm{O}-\mathrm{Br}$ bond breaking in highly excited vibrations of $\mathrm{HOBr}$ molecules. It is known that photolysis of the refrigerator refrigerant Freon produces bromine $(\mathrm{Br})$, which leads to the destruction of atmospheric $\mathrm{O}_{3}$ [1]. It has been shown that $\mathrm{OH}$ radicals and halogen atoms are the main sources of the formation of photochemical smog, and, therefore, the study of the dynamical properties of $\mathrm{HOBr}$ molecules in highly excited vibration has attracted a lot of attention.

The dynamics of bending and $\mathrm{OBr}$ stretching vibration of $\mathrm{HOBr}$ molecules have already been fully studied, and the main research methods are the first principle calculations or semi-classical methods [2-9]. Compared with the first-principle calculation, the semi-classical method can provide a more intuitive physical image and avoid the tedious calculations. Fruitful achievements of the research of highly excited vibration have been obtained by semi-classical methods, and some important conclusions provide a way to understand molecular dynamics characteristics [6,7]. In recent years, a new semi-classical method "dynamics potential" [5-7] has been proposed and applied to study highly excited vibrational molecular states. Applications of a dynamical potential phase and space analysis could both verify the conclusion of the first-principle method and give detailed physical pictures, including molecular isomerization [10], chaotic dynamics, dissociation dynamics [11-13] and other information.

In this work, the dynamic potentials of highly excited vibrational states of $\mathrm{HOBr}$ in bending and of $\mathrm{O}-\mathrm{Br}$ stretching coordinates with anharmonic resonance and Fermi coupling will be shown. The effect of the $\mathrm{H}-\mathrm{O}$ stretching vibration mode on the $\mathrm{O}-\mathrm{Br}$ stretching mode and the $\mathrm{H}-\mathrm{O}-\mathrm{Br}$ bending mode under different Polyad numbers will be investigated and the dynamical potentials, including the corresponding phase space trajectories will also be studied, which is helpful to understand the dynamics of highly excited vibrational states.

\section{The Semi-Classical Hamiltonian of the HOBr System}

The dynamical properties of highly excited vibrational states of $\mathrm{HOBr}$ in the energy region of $5 \times 10^{3}-2.5 \times 10^{4} \mathrm{~cm}^{-1}$ are abundant and are the focus of this work [2,9,13]. The HOBr vibration Hamiltonian in the energy region and its corresponding coefficient can be obtained [2,13] as follows:

$$
\begin{aligned}
H= & \omega_{1}\left(n_{1}+\frac{1}{2}\right)+\omega_{2}\left(n_{2}+\frac{1}{2}\right)+\omega_{3}\left(n_{3}+\frac{1}{2}\right) \\
& +x_{11}\left(n_{1}+\frac{1}{2}\right)^{2}+x_{12}\left(n_{1}+\frac{1}{2}\right)\left(n_{2}+\frac{1}{2}\right)+x_{13}\left(n_{1}+\frac{1}{2}\right)\left(n_{3}+\frac{1}{2}\right) \\
& +x_{22}\left(n_{2}+\frac{1}{2}\right)^{2}+x_{23}\left(n_{2}+\frac{1}{2}\right)\left(n_{3}+\frac{1}{2}\right)+x_{33}\left(n_{3}+\frac{1}{2}\right)^{2} \\
& +y_{113}\left(n_{1}+\frac{1}{2}\right)^{2}\left(n_{3}+\frac{1}{2}\right)+y_{122}\left(n_{1}+\frac{1}{2}\right)\left(n_{2}+\frac{1}{2}\right)^{2}+y_{133}\left(n_{1}+\frac{1}{2}\right)\left(n_{3}+\frac{1}{2}\right)^{2} \\
& +y_{223}\left(n_{2}+\frac{1}{2}\right)^{2}\left(n_{3}+\frac{1}{2}\right)+z_{1133}\left(n_{1}+\frac{1}{2}\right)^{2}\left(n_{3}+\frac{1}{2}\right)^{2}+z_{1333}\left(n_{1}+\frac{1}{2}\right)\left(n_{3}+\frac{1}{2}\right)^{3} \\
& +z_{2333}\left(n_{2}+\frac{1}{2}\right)\left(n_{3}+\frac{1}{2}\right)^{3}+z_{3333}\left(n_{3}+\frac{1}{2}\right)^{4}+z_{11112}\left(n_{1}+\frac{1}{2}\right)^{4}\left(n_{2}+\frac{1}{2}\right) \\
& +z_{11122}\left(n_{1}+\frac{1}{2}\right)^{3}\left(n_{2}+\frac{1}{2}\right)^{2}+z_{11222}\left(n_{1}+\frac{1}{2}\right)^{2}\left(n_{2}+\frac{1}{2}\right)^{3}+z_{11223}\left(n_{1}+\frac{1}{2}\right)^{2}\left(n_{2}+\frac{1}{2}\right)^{2}\left(n_{3}+\frac{1}{2}\right) \\
& +z_{12222}\left(n_{1}+\frac{1}{2}\right)\left(n_{2}+\frac{1}{2}\right)^{4}+z_{22233}\left(n_{2}+\frac{1}{2}\right)^{3}\left(n_{3}+\frac{1}{2}\right)^{2}+z_{22333}\left(n_{2}+\frac{1}{2}\right)^{2}\left(n_{3}+\frac{1}{2}\right)^{3} \\
& +\left[\mathrm{k}_{1}\left(n_{1}+\frac{1}{2}\right)+\mathrm{k}_{2} \mathrm{n}_{2}+\mathrm{k}_{3}\left(\mathrm{n}_{3}+\frac{3}{2}\right)+\mathrm{k}_{22} \mathrm{n}_{2}^{2}\right]\left(\mathrm{a}_{2}^{+} \mathrm{a}_{3}^{2}+\mathrm{a}_{3}^{+2} \mathrm{a}_{2}\right)
\end{aligned}
$$


The corresponding coefficients are shown in Table 1, where the Subscripts 1, 2, and 3, respectively, correspond to the $\mathrm{H}-\mathrm{O}$ stretching vibration mode, the mode of the bending vibration between the angle of $\mathrm{H}-\mathrm{O}$ and $\mathrm{O}-\mathrm{Br}$, and the $\mathrm{O}-\mathrm{Br}$ bond stretching vibration mode. The values $a^{+}$and $a$ are the creation and the destruction operators, which represent the increase or decrease of the vibration mode corresponding to the quantum number. The value $n$ indicates the quantum number of the vibration modes (for convenience, hereinafter we also use $n$ to denote the corresponding vibration modes, which will be indicated with a $q$ in the position coordinates and the momentum coordinates will be indicated with a $p$ ). The value $\omega$ is the corresponding harmonic vibration coefficient, while $x, y, z$ denote the nonlinear coupling coefficients among the different modes. Strong 2:1 Fermi resonance coupling exists between the $n_{2}$ and $n_{3}$ mode in $\mathrm{HOBr}$ and is observed in reference [2,3], and the strength of this resonance is related to the quantum numbers of the three modes. Based on the above-mentioned reasons, $\mathrm{k}$ represents the Fermi resonance strength coefficient in Hamiltonian between the bending vibration and $\mathrm{O}-\mathrm{Br}$ stretching vibration.

Table 1. The coefficient of $\mathrm{HOBr}$ molecular vibration Hamiltonian.

\begin{tabular}{cccc}
\hline Parameter name & $\begin{array}{c}\text { Parameter values } \\
\left(\mathbf{c m}^{-\mathbf{1}}\right)\end{array}$ & Parameter name & $\begin{array}{c}\text { Parameter values } \\
\left(\mathbf{c m}^{-\mathbf{1}}\right)\end{array}$ \\
\hline$\omega_{1}$ & 3769.5381 & $z_{1333}$ & 0.0121 \\
$\omega_{2}$ & 1187.7106 & $z_{2333}$ & -0.0015 \\
$\omega_{3}$ & 622.3722 & $z_{3333}$ & -0.0006 \\
$x_{11}$ & -70.7877 & $z_{11112}$ & 0.3505 \\
$x_{12}$ & -26.8651 & $z_{11122}$ & -0.6569 \\
$x_{13}$ & 10.3315 & $z_{11222}$ & 0.2578 \\
$x_{22}$ & -5.4432 & $z_{11223}$ & 0.0326 \\
$x_{23}$ & -3.7702 & $z_{12222}$ & -0.0202 \\
$x_{33}$ & -3.5507 & $z_{22233}$ & 0.0033 \\
$y_{113}$ & -3.4216 & $z_{22333}$ & -0.0017 \\
$y_{122}$ & -1.1955 & $k_{1}$ & 0.8717 \\
$y_{133}$ & -0.9150 & $k_{2}$ & -0.3183 \\
$y_{223}$ & -0.6071 & $k_{3}$ & -0.1935 \\
$z_{1133}$ & 0.2036 & $k_{22}$ & -0.0170 \\
\hline
\end{tabular}

Besides the conserved quantities of the $n_{1}$, considering the 2:1 Fermi resonance, $2 n_{2}+n_{3}$ is also a conserved quantity as a whole, which is called the Polyad number ( $\mathrm{P}$ number). It is known that Equation (1) is available to study the dynamical properties of highly excited vibrational states of $\mathrm{HOBr}$ in the region of $n_{1} \leq 7, P \leq 31$ [9].

It is also easy to semi-classify the second quantization Hamiltonian (1), which is useful in the further analysis. The coset space $\mathrm{SU}(2) / \mathrm{U}(1)$ [14] can be used as the representing space of $\mathrm{HOBr}$ 's Hamiltonian, so it can be rewritten using the bending coordinates $\left(q_{2}, p_{2}\right)$ as follows: 


$$
\begin{aligned}
H( & \left.n_{1}, q_{2}, p_{2}, P\right)=\omega_{1}\left(n_{1}+\frac{1}{2}\right)+\omega_{2}\left(\frac{p_{2}^{2}+q_{2}^{2}}{2}+\frac{1}{2}\right)+\omega_{3}\left(P-\left(p_{2}^{2}+q_{2}^{2}\right)+\frac{1}{2}\right)+X_{11}\left(n_{1}+\frac{1}{2}\right)^{2} \\
& +X_{12}\left(n_{1}+\frac{1}{2}\right)\left(\frac{p_{2}^{2}+q_{2}^{2}}{2}+\frac{1}{2}\right)+X_{13}\left(n_{1}+\frac{1}{2}\right)\left(P-\left(p_{2}^{2}+q_{2}^{2}\right)+\frac{1}{2}\right)+X_{22}\left(\frac{p_{2}^{2}+q_{2}^{2}}{2}+\frac{1}{2}\right)^{2} \\
& +X_{23}\left(\frac{p_{2}^{2}+q_{2}^{2}}{2}+\frac{1}{2}\right)\left(P-\left(p_{2}^{2}+q_{2}^{2}\right)+\frac{1}{2}\right)+X_{33}\left(P-\left(p_{2}^{2}+q_{2}^{2}\right)+\frac{1}{2}\right)^{2} \\
& +y_{113}\left(n_{1}+\frac{1}{2}\right)^{2}\left(P-\left(p_{2}^{2}+q_{2}^{2}\right)+\frac{1}{2}\right)+y_{122}\left(n_{1}+\frac{1}{2}\right)\left(\frac{p_{2}^{2}+q_{2}^{2}}{2}+\frac{1}{2}\right)^{2} \\
& +y_{133}\left(n_{1}+\frac{1}{2}\right)\left(P-\left(p_{2}^{2}+q_{2}^{2}\right)+\frac{1}{2}\right)^{2}+y_{223}\left(\frac{p_{2}^{2}+q_{2}^{2}}{2}+\frac{1}{2}\right)^{2}\left(P-\left(p_{2}^{2}+q_{2}^{2}\right)+\frac{1}{2}\right) \\
& +z_{1133}\left(n_{1}+\frac{1}{2}\right)^{2}\left(P-\left(p_{2}^{2}+q_{2}^{2}\right)+\frac{1}{2}\right)^{2}+z_{1333}\left(n_{1}+\frac{1}{2}\right)\left(P-\left(p_{2}^{2}+q_{2}^{2}\right)+\frac{1}{2}\right)^{3} \\
& +z_{2333}\left(\frac{p_{2}^{2}+q_{2}^{2}}{2}+\frac{1}{2}\right)\left(P-\left(p_{2}^{2}+q_{2}^{2}\right)+\frac{1}{2}\right)^{3}+z_{3333}\left(P-\left(p_{2}^{2}+q_{2}^{2}\right)+\frac{1}{2}\right)^{4} \\
& +z_{11112}\left(n_{1}+\frac{1}{2}\right)^{4}\left(\frac{p_{2}^{2}+q_{2}^{2}}{2}+\frac{1}{2}\right)+z_{11122}\left(n_{1}+\frac{1}{2}\right)^{3}\left(\frac{p_{2}^{2}+q_{2}^{2}}{2}+\frac{1}{2}\right)^{2} \\
& +z_{11222}\left(n_{1}+\frac{1}{2}\right)^{2}\left(\frac{p_{2}^{2}+q_{2}^{2}}{2}+\frac{1}{2}\right)^{3}+z_{11223}\left(n_{1}+\frac{1}{2}\right)^{2}\left(\frac{p_{2}^{2}+q_{2}^{2}}{2}+\frac{1}{2}\right)^{2}\left(P-\left(p_{2}^{2}+q_{2}^{2}\right)+\frac{1}{2}\right) \\
& +z_{12222}\left(n_{1}+\frac{1}{2}\right)\left(\frac{p_{2}^{2}+q_{2}^{2}}{2}+\frac{1}{2}\right)^{4}+z_{22233}\left(\frac{p_{2}^{2}+q_{2}^{2}}{2}+\frac{1}{2}\right)^{3}\left(P-\left(p_{2}^{2}+q_{2}^{2}\right)+\frac{1}{2}\right)^{2} \\
& +z_{22333}\left(\frac{p_{2}^{2}+q_{2}^{2}}{2}+\frac{1}{2}\right)^{2}\left(P-\left(p_{2}^{2}+q_{2}^{2}\right)+\frac{1}{2}\right)^{3} \\
& +\left[\mathrm{k}_{1}\left(n_{1}+\frac{1}{2}\right)+\mathrm{k}_{2}\left(\frac{p_{2}^{2}+q_{2}^{2}}{2}\right)+\mathrm{k}_{3}\left(P-\left(p_{2}^{2}+q_{2}^{2}\right)+\frac{3}{2}\right)+\mathrm{k}_{22}\left(\frac{p_{2}^{2}+q_{2}^{2}}{2}\right)^{2}\right] \sqrt{2}\left(P-\left(p_{2}^{2}+q_{2}^{2}\right)\right) q_{2}
\end{aligned}
$$

With the coordinates $\left(q_{3}, p_{3}\right)$, the Hamiltonian can be written as:

$$
\begin{aligned}
H( & \left.n_{1}, q_{3}, p_{3}, P\right)=\omega_{1}\left(n_{1}+\frac{1}{2}\right)+\omega_{2}\left(\frac{P}{2}-\frac{p_{3}^{2}+q_{3}^{2}}{4}+\frac{1}{2}\right)+\omega_{3}\left(\frac{p_{3}^{2}+q_{3}^{2}}{2}+\frac{1}{2}\right)+X_{11}\left(n_{1}+\frac{1}{2}\right)^{2} \\
& +X_{12}\left(n_{1}+\frac{1}{2}\right)\left(\frac{P}{2}-\frac{p_{3}^{2}+q_{3}^{2}}{4}+\frac{1}{2}\right)+X_{13}\left(n_{1}+\frac{1}{2}\right)\left(\frac{p_{3}^{2}+q_{3}^{2}}{2}+\frac{1}{2}\right)+X_{22}\left(\frac{P}{2}-\frac{p_{3}^{2}+q_{3}^{2}}{4}+\frac{1}{2}\right)^{2} \\
& +X_{23}\left(\frac{P}{2}-\frac{p_{3}^{2}+q_{3}^{2}}{4}+\frac{1}{2}\right)\left(\frac{p_{3}^{2}+q_{3}^{2}}{2}+\frac{1}{2}\right)+X_{33}\left(\frac{p_{3}^{2}+q_{3}^{2}}{2}+\frac{1}{2}\right)^{2} \\
& +y_{113}\left(n_{1}+\frac{1}{2}\right)^{2}\left(\frac{p_{3}^{2}+q_{3}^{2}}{2}+\frac{1}{2}\right)+y_{122}\left(n_{1}+\frac{1}{2}\right)\left(\frac{P}{2}-\frac{p_{3}^{2}+q_{3}^{2}}{4}+\frac{1}{2}\right)^{2} \\
& +y_{133}\left(n_{1}+\frac{1}{2}\right)\left(\frac{p_{3}^{2}+q_{3}^{2}}{2}+\frac{1}{2}\right)^{2}+y_{223}\left(\frac{P}{2}-\frac{p_{3}^{2}+q_{3}^{2}}{4}+\frac{1}{2}\right)^{2}\left(\frac{p_{3}^{2}+q_{3}^{2}}{2}+\frac{1}{2}\right) \\
& +z_{1133}\left(n_{1}+\frac{1}{2}\right)^{2}\left(\frac{p_{3}^{2}+q_{3}^{2}}{2}+\frac{1}{2}\right)^{2}+z_{1333}\left(n_{1}+\frac{1}{2}\right)\left(\frac{p_{3}^{2}+q_{3}^{2}}{2}+\frac{1}{2}\right)^{3} \\
& +z_{2333}\left(\frac{P}{2}-\frac{p_{3}^{2}+q_{3}^{2}}{4}+\frac{1}{2}\right)\left(\frac{p_{3}^{2}+q_{3}^{2}}{2}+\frac{1}{2}\right)^{3}+z_{3333}\left(\frac{p_{3}^{2}+q_{3}^{2}}{2}+\frac{1}{2}\right)^{4} \\
& +z_{11112}\left(n_{1}+\frac{1}{2}\right)^{4}\left(\frac{P}{2}-\frac{p_{3}^{2}+q_{3}^{2}}{4}+\frac{1}{2}\right)+z_{11122}\left(n_{1}+\frac{1}{2}\right)^{3}\left(\frac{P}{2}-\frac{p_{3}^{2}+q_{3}^{2}}{4}+\frac{1}{2}\right)^{2} \\
& +z_{11222}\left(n_{1}+\frac{1}{2}\right)^{2}\left(\frac{P}{2}-\frac{p_{3}^{2}+q_{3}^{2}}{4}+\frac{1}{2}\right)^{3}+z_{11223}\left(n_{1}+\frac{1}{2}\right)^{2}\left(\frac{P}{2}-\frac{p_{3}^{2}+q_{3}^{2}}{4}+\frac{1}{2}\right)^{2}\left(\frac{p_{3}^{2}+q_{3}^{2}}{2}+\frac{1}{2}\right) \\
& +z_{12222}\left(n_{1}+\frac{1}{2}\right)\left(\frac{P}{2}-\frac{p_{3}^{2}+q_{3}^{2}}{4}+\frac{1}{2}\right)^{4}+z_{22233}\left(\frac{P}{2}-\frac{p_{3}^{2}+q_{3}^{2}}{4}+\frac{1}{2}\right)^{3}\left(\frac{p_{3}^{2}+q_{3}^{2}}{2}+\frac{1}{2}\right)^{2} \\
& +z_{22333}\left(\frac{P}{2}-\frac{p_{3}^{2}+q_{3}^{2}}{4}+\frac{1}{2}\right)^{2}\left(\frac{p_{3}^{2}+q_{3}^{2}}{2}+\frac{1}{2}\right)^{3} \\
& +\left[\mathrm{k}_{1}\left(n_{1}+\frac{1}{2}\right)+\mathrm{k}_{2}\left(\frac{P}{2}-\frac{p_{3}^{2}+q_{3}^{2}}{4}\right)+\mathrm{k}_{3}\left(\frac{p_{3}^{2}+q_{3}^{2}}{2}+\frac{3}{2}\right)+\mathrm{k}_{22}\left(\frac{P}{2}-\frac{p_{3}^{2}+q_{3}^{2}}{4}\right)^{2}\right] \sqrt{\frac{P}{2}-\frac{p_{3}^{2}+q_{3}^{2}}{4}}\left(q_{3}^{2}-p_{3}^{2}\right) \\
&
\end{aligned}
$$


With the semi-classical Hamiltonian, the above-mentioned can further be used to obtain the dynamics potential and, in this way, we can study the dynamic nature of the HOBr's highly excited vibrational states.

\section{The Dynamics Potential of Highly Excited Vibration of $\mathrm{HOBr}$}

The dynamical potential of $H\left(q_{i}, p_{i}, P\right)$ is the effective environment in which the $q_{i}$ coordinate stays for each $\mathrm{P}$ in a certain molecule. This is achieved by calculating the maximal and minimal energies by varying $p_{i}$ for each $q_{i}$ under the condition that $\mathrm{n}_{2}$ and $\mathrm{n}_{3}$ are nonnegative. The dynamical potential composed of these maximal and minimal energies as a function of $q_{i}$ is represented by a closed curve in which the quantal levels are enclosed. The dynamical potential also defines the $q_{i}$ region for each level it encloses. Furthermore, the points in the dynamical potential corresponding to $\partial H / \partial q_{i}=0$ are fixed points in the dynamical space [5]. In this section, two main parts will be addressed as follows: (1) the dynamical potential influences of the $\mathrm{H}-\mathrm{O}$ stretching model on the $\mathrm{H}-\mathrm{O}-\mathrm{B}$ bending model and the $\mathrm{O}-\mathrm{Br}$ stretching model and (2) for instance, the phase space trajectories, the action integrals, and quantal environments of all energy levels in the dynamical potentials when $P$ is equal to 27 will be studied.

\subsection{The Study of Dynamical Potentials and Their Characteristics of Different Quantum Numbers $\mathrm{n}_{1}$ Corresponding to Typical Polyad Numbers in $\mathrm{HOBr}$}

The dynamical potentials of $\mathrm{HOBr}$ (in coordinates $q_{2}, q_{3}$,) with $n_{1}=0,1,2,3$ of different $\mathrm{P}$ numbers is considered, and their levels can be calculated with the aforementioned Hamiltonian (ground state from the potential energy at the bottom of $2764.0 \mathrm{~cm}^{-1}$ ). It is found that the dynamical potentials are different for small and large $P$ numbers. The dynamical potential is shown as follows (the path marked by the fixed point is in accordance with the literature [13]) when $P$ is equal to 15 :

Figure 1 shows that when $n_{1}$ is equal to $0,1,2,3$, the dynamical potentials in the $q_{2}$ coordinates are all simple inverse Morse potentials, which shows that the $\mathrm{n}_{1}$ mode has little effect on the dynamical potentials of the $q_{2}$ coordinates. In the theory of the dynamical potential [9] it is noted that, for an inverse Morse potential, the stability of the lowest energy level is the worst in the inverted Morse potential corresponding to a certain $P$, which indicates that $\mathrm{H}-\mathrm{O}$ stretching has no influence on the stability of the energy level corresponding to the $\mathrm{H}-\mathrm{O}-\mathrm{Br}$ bending vibration mode under the same $P$ number. In the sense of the geometrical shape of the dynamical potential, it is found that the top of dynamical potentials only gradually flattens with the increase of $n_{1}$, which shows that the $n_{1}$ mode has almost no significant effect on the coupling of $n_{2}$ and $n_{3}$. In contrast, the dynamical potentials of $\mathrm{HOBr}$ in the $q_{3}$ coordinate consist of an inverted Morse potential and a positive Morse potential, and with the increase of $n_{1}$, the top of the inverted Morse potential gradually flattens and the positive Morse potential wells become deeper, which shows that the dynamical potential is turning into an almost pure positive Morse potential, especially when $n_{1}$ is equal to three. As a result, the stability of the high energy levels becomes worse while the original fixed-point still remains the same. All the above phenomena show that, although the $\mathrm{n}_{1}$ mode is not coupled with the $n_{2}$ and $n_{3}$, it still has some effect on the resonant coupling of the other two modes, and this impact is reflected in the stability of the stretching mode (when $n_{1}$ is equal to three, this impact is particularly evident). Or said differently, the 
$\mathrm{H}-\mathrm{O}$ stretching mode has an important influence on the dynamics of the other degrees of freedom. According to qualitative analysis, this is because the intramolecular vibrational relaxation (IVR), caused by the resonance between $n_{2}$ and $n_{3}$, which enhances the stability of the energy levels [9], is weakened in the high-energy region. In the previous literature, the uncoupling modes in molecules have hardly ever been considered in the study of molecular dynamical behavior, and in this work, it is shown that the previous cognition maybe not comprehensive [2,3].

Figure 1. The dynamical potentials of $\operatorname{HOBr}(P=15)$ with $n_{1}=0,1,2,3$. The energy levels included in the $\mathrm{P}$ number are represented by the lines.
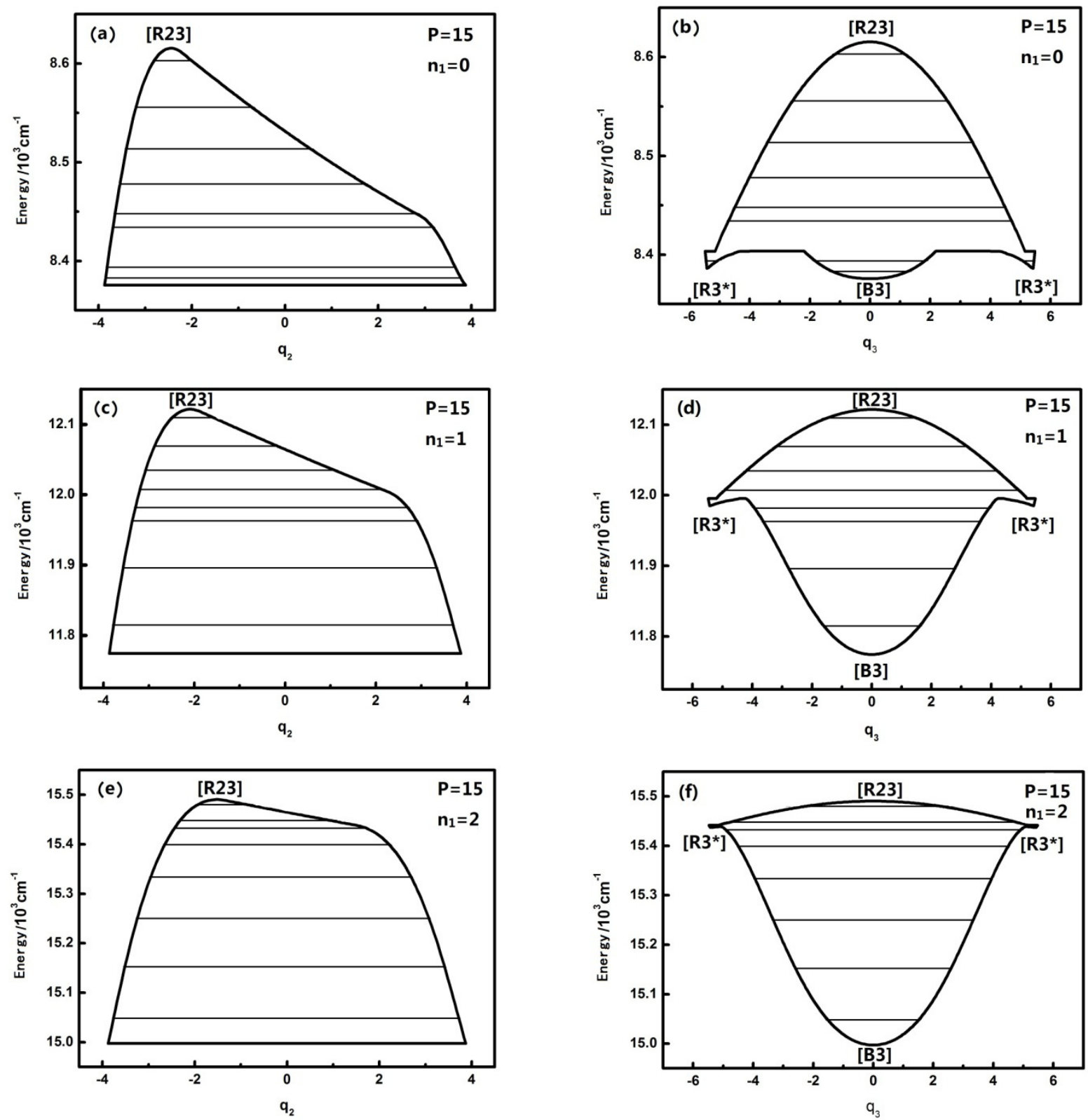
Figure 1. Cont.
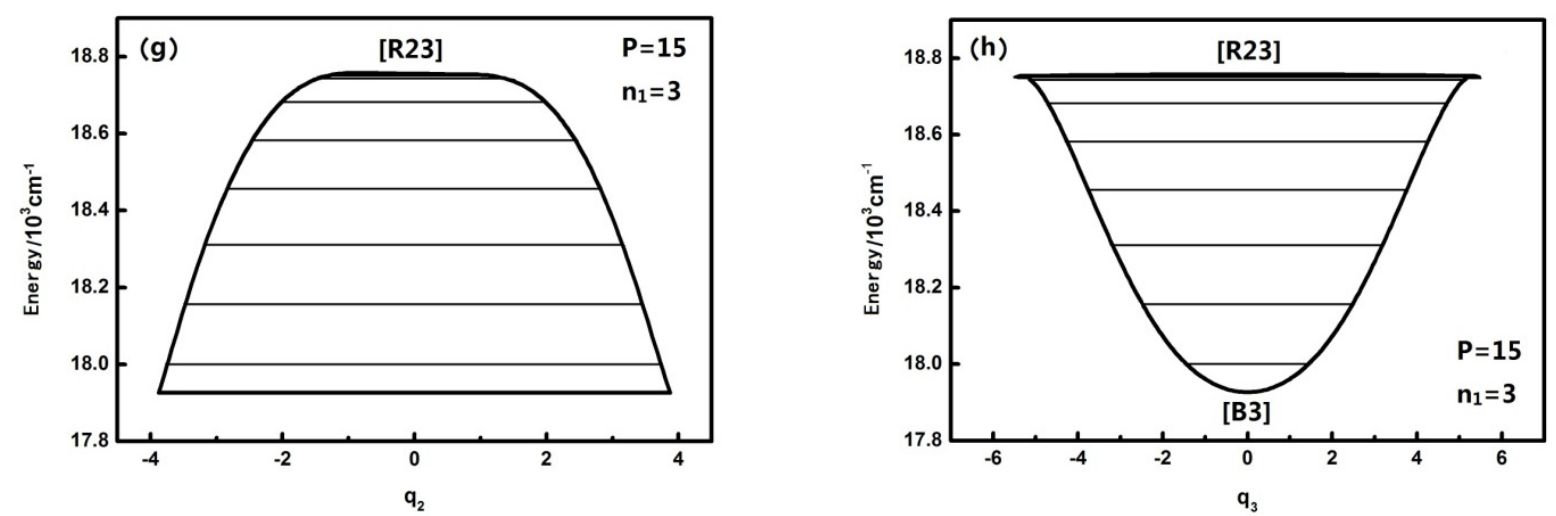

For larger $P$ numbers, the change of the dynamical potentials is much more complicated. The dynamical potentials are shown as follows when $P$ is, for instance, equal to 27 :

When $n_{1}$ is equal to three, the geometrical shape of the dynamical potentials of both $q_{2}$ and $q_{3}$ becomes increasingly complex and the number of the potential wells also increases. There are two inverted Morse in the $q_{2}$ dynamical potentials, yet there are two positive Morse in the $q_{3}$ dynamical potentials. This shows that with the increase of the $n_{1}$ mode, the dynamical potential system consists of $n_{2}$ and $n_{3}$, which makes it more complex. This conclusion confirms once again that the $\mathrm{n}_{1}$ mode has some effect on the dynamical behavior of the other mutually coupled resonance modes and that this effect is more apparent compared with the case of $P=15$, which shows that the uncoupling mode becomes much more important to the molecular dynamics behaviors with Polyad number.

Figure 2 also shows that the $q_{2}$ dynamical potential with $n_{1}=3$ is more complex than the one with $n_{1}=0,1,2$ and that the $\left[B_{2}\right]$ fixed point appears. This fixed point leads to the vibration modes corresponding to the highest three energy levels in the dynamical potential becoming localized vibrational when $n_{1}$ is equal to three (in the two inverted Morse potentials). In addition, the fixed points of the dynamical potentials in $q_{3}$ remain the same, but when $n_{1}$ is equal to three, the vibration mode corresponding to the low energy level becomes localized vibrational (in the two positive Morse potentials). Compared with the dynamical potentials when $\mathrm{P}$ is equal to 15 , it is obvious that the effect of $\mathrm{n}_{1}$ on $n_{2}$ (or $n_{3}$ 's resonant coupling) is closely related to the value of the $\mathrm{P}$ number. The general conclusion is that $\mathrm{n}_{1}$ has little effect on the geometrical shape of the dynamical potential of $q_{2}, q_{3}$ when the $\mathrm{P}$ number is small, however, this effect becomes larger when the $P$ number is larger.

In the above-mentioned study, it was found that although the $\mathrm{H}-\mathrm{O}$ stretching mode was not coupled with the $\mathrm{H}-\mathrm{O}-\mathrm{Br}$ bending mode and the $\mathrm{O}-\mathrm{Br}$ stretching mode, it still affected the resonant coupling of the two modes, thereby affecting the dynamics of HOBr. It is elucidated with qualitative analysis that the geometrical shape of the dynamical potentials and the corresponding fixed points are sensitive to the change of the $\mathrm{H}-\mathrm{O}$ stretching mode in the sense of geometry. However, this phenomenon and the related explanation should be verified in further studies of other molecular systems. 
Figure 2. The dynamical potentials of $\operatorname{HOBr}(P=27)$ with $n_{1}=0,1,2,3$. The energy levels included in the $\mathrm{P}$ number are represented by the lines.
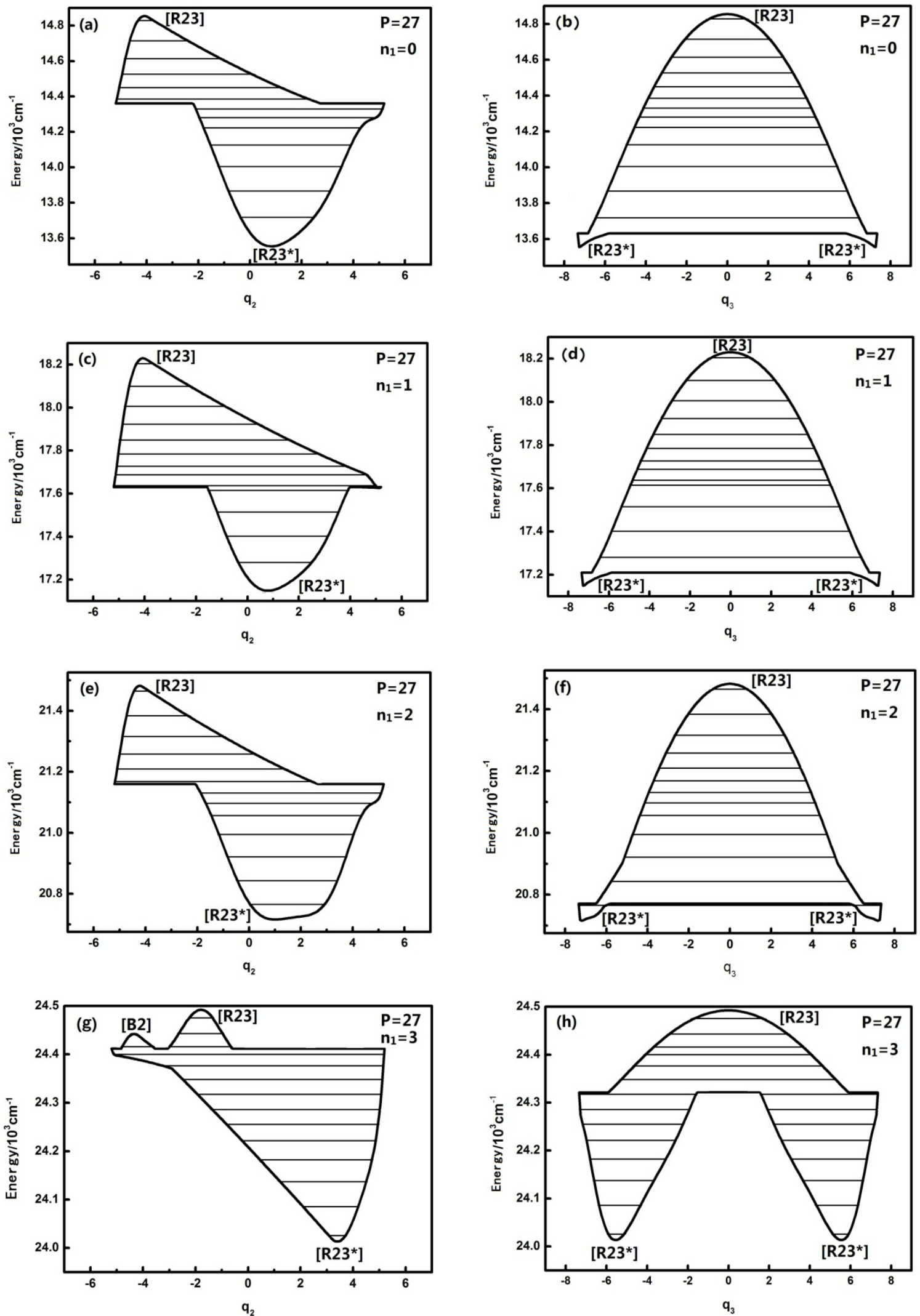


\subsection{The Nature and Quantal Environment of the Level of a Specific Polyad Number $(\mathrm{P}=27)$}

The qualitatively different quantum number $\mathrm{n}_{1}$ of the $\mathrm{HOBr}$ system corresponding to dynamical potentials and characteristics are discussed in the previous section. Next we will discuss quantitatively the dynamical potentials of the specific $P$ numbers with the analysis of the phase space trajectories and the action integral of every energy level. For instance, the dynamical potentials of $\mathrm{HOBr}$ with $P=27$ are shown in Figure 3a,b in $q_{2}$ and $q_{3}$ coordinates, where the horizontal lines show the energy levels sharing the designated $P$. The reason why the case of $P=27$ is singled out for discussion is that this case is quite representative and possesses the most fruitful characteristics. The dynamic potentials obtained when $n_{1}$ is equal to three are as follows:

Figure 3. The dynamical potentials in $q_{2}$ (a) and $q_{3}$ (b) coordinates for $\mathrm{HOBr}$ with $P=27$. The horizontal lines show the quantal states, L0-L13. [B], [R], [R*] are the stable and unstable fixed points, respectively.
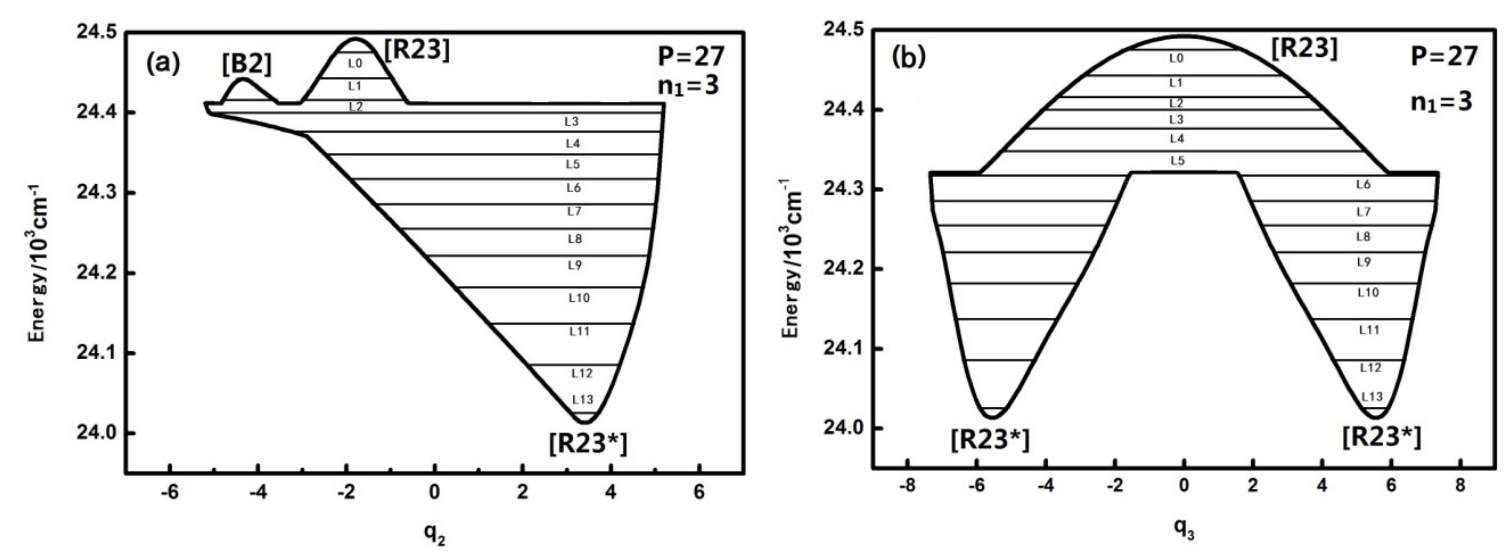

The energy is with respect to the ground state which is $2764.0 \mathrm{~cm}^{-1}$ above the bottom of the potential well, and the points in Figure 3 designated by $[\mathrm{B}],[\mathrm{R}],\left[\mathrm{R}^{*}\right]$ are stable fixed points where both $\partial H / \partial q_{i}$ and $\partial H / \partial p_{i}(i=2$ or 3) are zero [10-13]. It is noted that [R23] is stable for the upper realm of the dynamical potential because the potential curve is inverse (upside down) and low energy regions have a positive potential at the bottom, which indicates that [R23*] is a stable fixed point. Similarly, in Figure 3a, [B2] is also a stable fixed point. Generally, dynamical potentials are considered to be able to find all of the fixed points by the two coordinates, and it is easy to find fixed points from intuitive geometry with the dynamics potential, which provides a convenient way to study the dynamics of a system.

To further quantitatively analyze the features of related energy levels, the representative trajectories of phase space in $p_{i}-q_{i}$ of each energy level is obtained. From the geometric properties of the pattern, the trajectory of phase space can be classified into different sets. It is found that the trajectories of L0-L1, L2, L3, L4-L8, L9-L13 in $q_{2}$ coordinates constitute a class and the ones of the L0-L1, L2, L3, L4-L6, L7-L13 corresponding levels, respectively, constitute a class in $q_{3}$ coordinates. They are partly shown in Figures 4 and 5: 
Figure 4. The trajectories of phase space of L1, L2, L3, L5, L7, L9 ( $q_{2}$ coordinates).
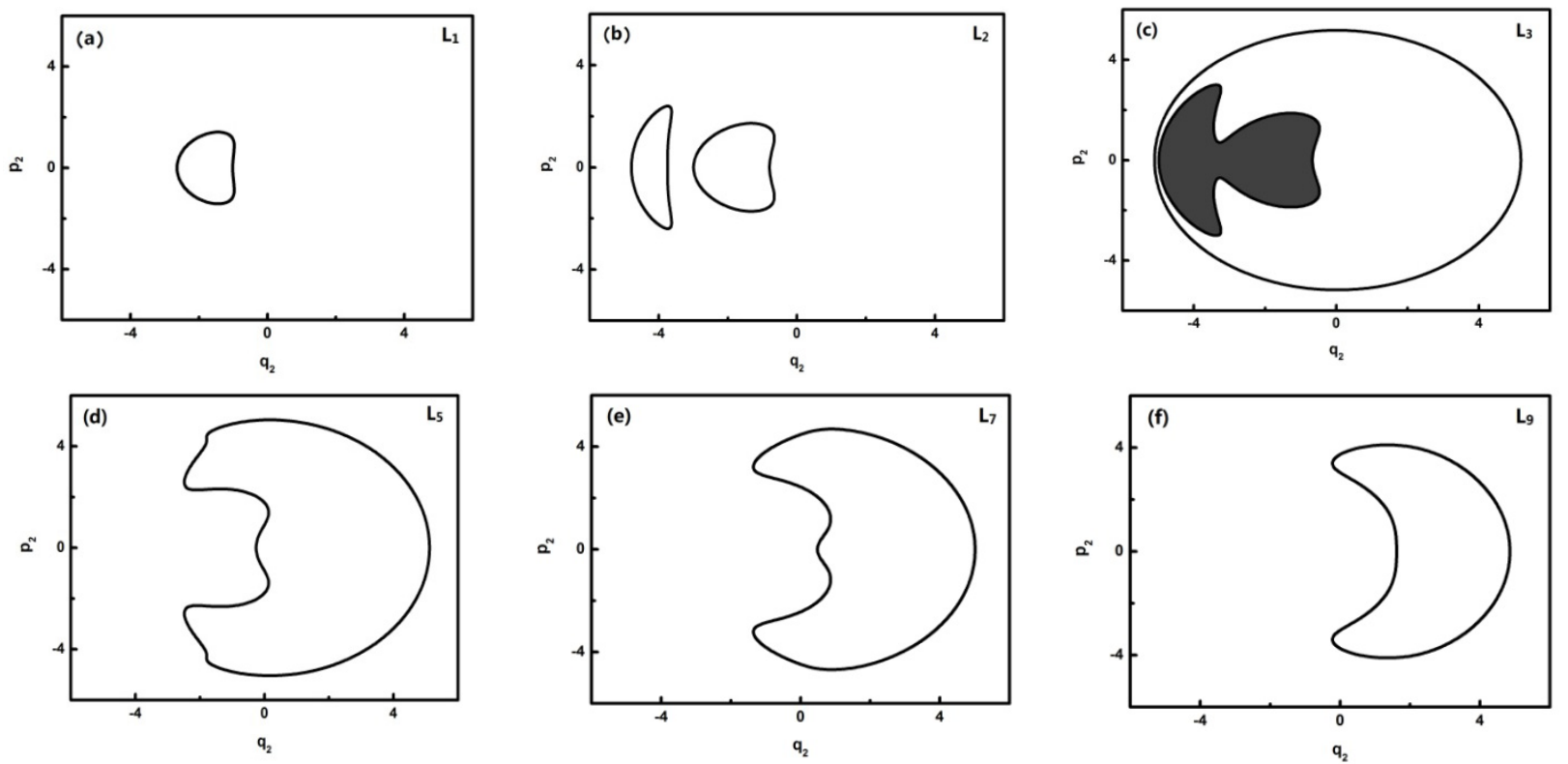

Figure 5. The trajectories of phase space of L1, L2, L3, L5, L7, L9 ( $q_{3}$ coordinates).
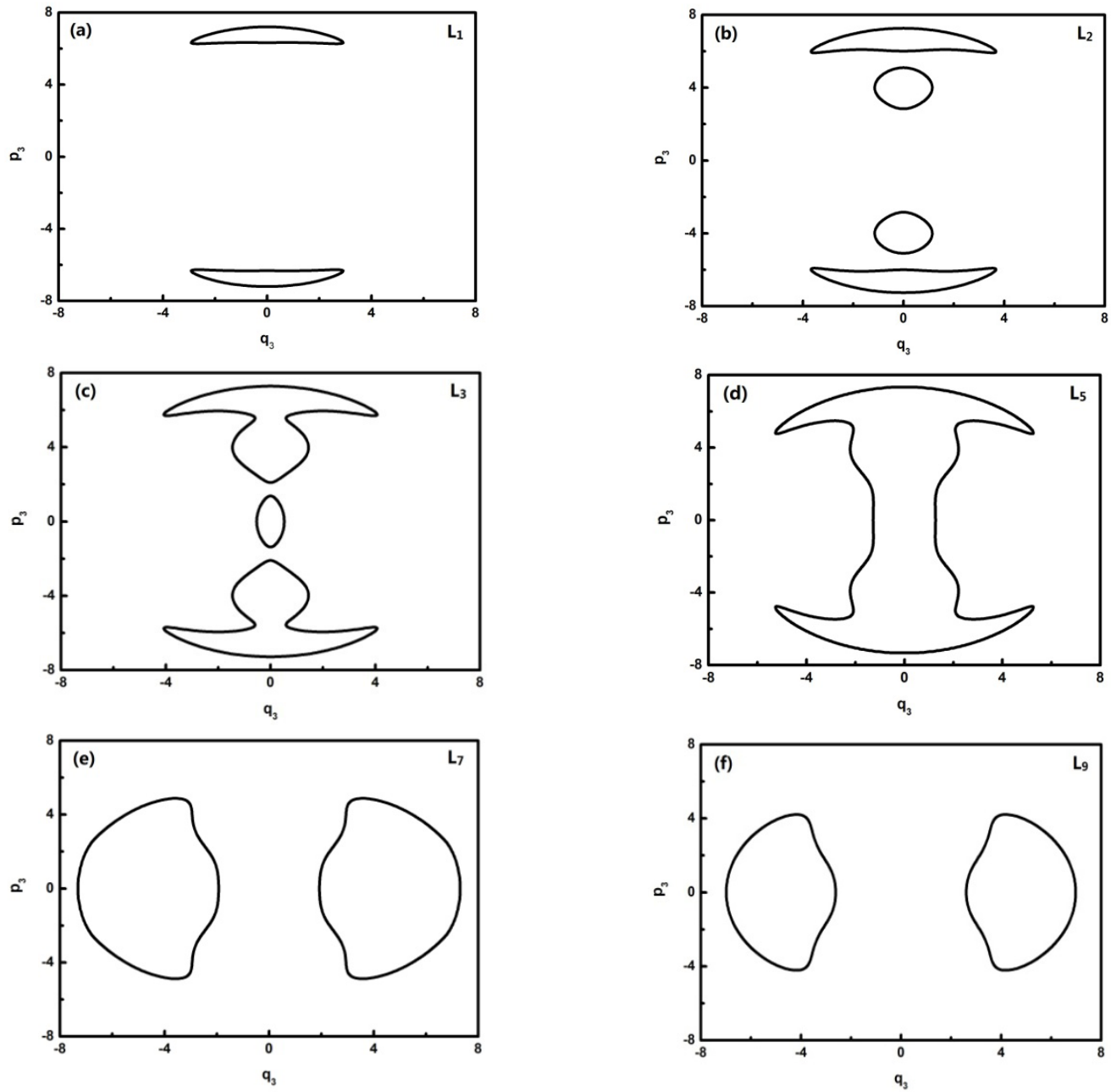
In Figure 4, L0-L2 are in inverted Morse potentials and the area of the trajectory of the phase space increases with the reduction of energy. L3-L13 are in a positive Morse potential and the area of the trajectory of the phase space decreases with the reduction of energy. It is also found that the trajectory of the phase space of L2 is divided into two separate trajectories, of which L2 is located at the double-well potential in the dynamical potential shown in Figure 3.

In Figure 5, L0-L6 are in inverted Morse potentials and the area of the phase diagram increases with the reduction of energy. L7-L13 are in the positive Morse potential and the area of the phase diagram decreases with the reduction of energy. The trajectories of the phase space of L0-L1 are composed of two symmetrical parts (the upper one and lower one) because there is a positive and negative direction of momentum, and particularly, the trajectory of the phase space of L2 is composed of four symmetrical parts due to a multiplied effect of positive/negative directions of momentum on the energy level located in the double-well potential (Figure 3a). There is some difference in the phase space of L3, and it is shown that the trajectory is composed of three parts caused by an approximate harmonic vibration. Similarly, there are two parts of the trajectory in the phase space of L7-L13 because they are located in the two potential wells. Through the above-mentioned analysis, we found that the trajectories of the phase space can more intuitively reflect the dynamical characteristics of the vibration mode.

In order to study the quantal environment of each energy level, the calculation of the action integral of the phase space trajectories (quantum number) is needed and the corresponding formula is as follows:

$$
\text { Action integral }=\frac{1}{2 \pi} \times \oint p_{i} d q_{i}
$$

The results are shown in Table 2:

Table 2. The action integrals of the levels corresponding to $P=27, n=3$.

\begin{tabular}{cccccc}
\hline $\begin{array}{c}\text { State } \\
\text { label }\end{array}$ & $\begin{array}{c}\text { Action integral } \\
\text { in }\left(\boldsymbol{q}_{\mathbf{2}}, \boldsymbol{p}_{\mathbf{2}}\right) \text { space }\end{array}$ & $\begin{array}{c}\text { Difference of the } \\
\text { action integrals the } \\
\text { neighboring levels }\end{array}$ & $\begin{array}{c}\text { State } \\
\text { label }\end{array}$ & $\begin{array}{c}\text { Action integral } \\
\text { in }\left(\boldsymbol{q}_{\mathbf{3}}, \boldsymbol{p}_{\mathbf{3}}\right) \text { space }\end{array}$ & $\begin{array}{c}\text { Difference of the } \\
\text { action integrals the } \\
\text { neighboring levels }\end{array}$ \\
\hline L0 & 0.20 & $/$ & L0 & 0.39 & $/$ \\
L1 & 0.61 & 0.41 & L1 & 1.21 & 0.82 \\
L2 & 1.64 & 1.03 & L2 & 3.27 & 2.06 \\
L3 & 10.83 & $/$ & L3 & 5.32 & 2.05 \\
L4 & 9.45 & 1.38 & L4 & 8.08 & 2.76 \\
L5 & 8.29 & 1.14 & L5 & 10.41 & 2.33 \\
L6 & 7.18 & 1.11 & L6 & 12.70 & 2.29 \\
L7 & 6.12 & 1.06 & L7 & 12.27 & $/$ \\
L8 & 5.13 & 0.99 & L8 & 10.27 & 2.00 \\
L9 & 4.19 & 0.94 & L9 & 8.37 & 1.90 \\
L10 & 3.19 & 1.00 & L10 & 6.39 & 1.98 \\
L11 & 2.19 & 1.00 & L11 & 4.37 & 2.02 \\
L12 & 1.18 & 1.01 & L12 & 2.37 & 2.00 \\
L13 & 0.19 & 0.99 & L13 & 0.37 & 2.00 \\
\hline
\end{tabular}


Table 2 shows the action integrals for the levels corresponding to $P=27$, calculated from the trajectories of the phase space in $\left(q_{2}, p_{2}\right)$ and $\left(q_{3}, p_{3}\right)$. From the action integrals calculated from $\left(q_{2}, p_{2}\right)$, the levels can be grouped into two subsets: L0 to L2 and L3 to L13. For the former ones, the action integral increases almost in a constant with the decline of energy levels due to the inverse-Morse well where they are located, and for the latter ones [15], the action integral decreases (also almost in a constant) with the decline of energy levels due to the positive Morse well where they are located. From the action integrals calculated from $\left(q_{3}, p_{3}\right)$, the levels can be grouped into two subsets, also with constant increment (decrement) in their action integrals: L0 to L6 and L7 to L13. For the former ones, the action integral increases with the decline of energy levels due to the inverse-Morse well where they are located, but with minor deviation on L2, and for the latter ones, the action integral decreases with the decline of energy levels due to the positive Morse wells where they are located. The constant action integral increment/decrement demonstrates the compatibility of our classical treatment with the quantized levels and also shows that the levels stay in various dynamical environments, which are defined essentially by the classical fixed points though these energies belong to the same Polyad number. In addition, from the view of the $\left(q_{2}, p_{2}\right)$ space, the difference between adjacent energy levels' action integrals is almost always the same (approximately equal to one, but the L1 is an exception and should be further studied in the future). This value is equal to two if in the view of $\left(q_{2}, p_{2}\right)$ because $P=2 n_{2}+n_{3}$, which is also shown in Table 2 . From the above results, it is elucidated that the action integral differential of adjacent energy levels nearly doubles that of $\left(q_{2}, p_{2}\right)$, which corresponds exactly with the system of 2:1 Fermi resonance. The dynamics potential is actually the same as the trajectory connecting each fixed point, depicting the restrictive conditions in which individual energy levels are located.

\section{Conclusions and Remarks}

The highly excited vibrational state is hard to study using the quantum mechanical calculation because of its nonlinear interactions, but it is full of important information. The algebraic Hamiltonian, action integral and dynamical potential can bring direct physical pictures into the geometric sense of this research field. It is well known that the effective Hamiltonians with a single resonant interaction only are completely integrable systems and that the quantization can be done by the quantization of action integrals $[16,17]$. However, as an alternative method, the dynamical potential can lead to most inferences being drawn by the quantal wave function algorithm. The subtle behavior of the state wave functions that show a drastic change of mode character in a set of states sharing a common Polyad number is found to be related to the unstable region/fixed point in the dynamical space. This approach is global in the sense that the focus is on a set of levels instead of individual ones, which is different from traditional semi-classical methods, but, nevertheless, it should be noted that our approach is but one branch in the field of algebraic approaches to molecular vibrational dynamics. With the methods mentioned above using $\mathrm{HOBr}$, it is not difficult to extend our subjects to other integrable and nonintegrable systems (such as $\mathrm{O}_{3}, \mathrm{NO}_{2}, \mathrm{CS}_{2}, \mathrm{C}_{2} \mathrm{H}_{2}$ and so on), including their dissociation, isomerization and dynamical symmetry. These conclusions are helpful and significant for us to understand the dynamics of molecular highly excited vibrational dynamics. 


\section{Acknowledgments}

This work was supported by the Natural Science Foundation of China (Grant No. 11104156), and the Natural Science Foundation of Jiangxi Province (Grant No. 2009GZW0001), the Open Foundation of Engineering Research Center of Nuclear Technology Application, Ministry of Education (Grant No. HJSJYB2011-05).

\section{Conflict of Interest}

The authors declare no conflict of interest.

\section{References}

1. Leu, M.T. Heterogeneous reactions of $\mathrm{N} 2 \mathrm{O} 5$ with $\mathrm{H} 2 \mathrm{O}$ and hcl on ice surfaces-implications for antarctic ozone depletion. Geophys. Res. Lett. 1988, 15, 851-854.

2. Jost, R.; Joyeux, M.; Skokov, S.; Bowman, J. Vibrational analysis of $\mathrm{HOCl}$ up to $98 \%$ of the dissociation energy with a Fermi resonance Hamiltonian. J. Chem. Phys. 1999, 111, 6807-6820.

3. Peterson, K.A.; Skokov, S.; Bowman, J. A theoretical study of the vibrational energy spectrum of the $\mathrm{HOCl} / \mathrm{HClO}$ system on an accurate ab initio potential energy surface. J. Chem. Phys. 1999, $111,7446-7456$.

4. Peterson, K.A. An accurate global ab initio potential energy surface for the $\mathrm{X}(1) \mathrm{A}^{\prime}$ electronic state of HOBr. J. Chem. Phys. 2000, 113, 4598-4612.

5. Joyeux, M.; Sugny, D.; Lombardi, M.; Jost, R.; Schinke, R.; Skokov, S.; Bowman, J. Vibrational dynamics up to the dissociation threshold: A case study of two-dimensional HOCl. J. Chem. Phys. 2000, 113, 9610-9621.

6. Weiss, J.; Hauschildt, J.; Grebenshchikov, S.Y.; Duren, R.; Schinke, R.; Koput, J.; Stamatiadis, S.; Farantos, S.C. Saddle-node bifurcations in the spectrum of HOCl. J. Chem. Phys. 2000, 112, 77-79.

7. Joyeux, M.; Grebenshchikov, S.Y.; Bredenbeck, J.; Schinke, R.; Farantos, S.C. Phase Space Geometry of Multi-dimensional Dynamical Systems and Reaction Processes. Geometric Structures of Phase Space in Multidimensional Chaos: Applications to Chemical Reaction Dynamics in Complex Systems, Volume 130; John Wiley \& Sons: Hoboken, NJ, USA, 2005.

8. Joyeux, M.; Farantos, S.C.; Schinke, R. Highly excited motion in molecules: Saddle-node bifurcations and their fingerprints in vibrational spectra. J. Chem. Phys. 2002, 106, 5407-5421.

9. Azzam, T.; Schinke, R.; Farantos, S.C.; Joyeux, M.; Peterson, K.A. The bound state spectrum of $\mathrm{HOBr}$ up to the dissociation limit: Evolution of saddle-node bifurcations. J. Chem. Phys. 2003, 118, 9643-9652.

10. Fang, C.; Wu, G.Z. Dynamical similarity in the highly excited vibrations of HCP and DCP: The dynamical potential approach. Comp. Theor. Chem. 2009, 910, 141-147.

11. Fang, C.; Wu, G.Z. Dynamical potential approach to dissociation of H-C bond in HCO highly excited vibration. Chin. Phys. B 2009, 18, 130-135.

12. Fang, C.; Wu, G.Z. Global dynamical analysis of vibrational manifolds of $\mathrm{HOCl}$ and $\mathrm{HOBr}$ under anharmonicity and Fermi resonance: The dynamical potential approach. Chin. Phys. B 2010, 19, 010509:1-010509:10. 
13. Fang, C.; Wu, G.Z. Bending localization of nitrous oxide under anharmonicity and Fermi coupling: the dynamical potential approach. Chin. Phys. B 2010, 19, 110513:1-110513:6.

14. Zhang, W.M.; Feng, D.H.; Gilmore, R. Coherent states: Theory and some applications. Rev. Mod. Phys. 1990, 62, 867-927.

15. Biczysko, M.; Tarroni, R. Theoretical calculation of rovibronic energy levels and anharmonic resonances in the ground X (2)Pi state of $\mathrm{HCP}+$ and DCP+. Phys. Chem. Chem. Phys. 2002, 4, $708-715$

16. Kellman, M.E. Approximate constants of motion for vibrational-spectra of many-oscillator systems with multiple anharmonic resonances. J. Phys. Chem. 1990, 93, 6630-6635.

17. Laurence, E.F.; Gregory, S.E. PERTRUB: A program for calculating vibrational energies by generalized algebraic quantization. J. Phys. Chem. 1987, 86, 6270-6282.

(C) 2013 by the authors; licensee MDPI, Basel, Switzerland. This article is an open access article distributed under the terms and conditions of the Creative Commons Attribution license (http://creativecommons.org/licenses/by/3.0/). 